\title{
HSD10 disease, neonatal type
}

INSERM

\section{Source}

INSERM. (1999). Orphanet: an online rare disease and orphan drug data base. HSD10 disease, neonatal type. ORPHA:391457

HSD10 disease, neonatal type is the most severe form of HSD10 disease, a rare neurometabolic disorder. It is characterized by severe metabolic/lactic acidosis in the neonatal period, little psychomotor development, seizures and severe progressive hypertrophic cardiomyopathy. Hepatic involvement and coagulopathy are rare. The disease is fatal within the first months of life. 